\title{
Mastering the complexity: drug therapy optimization in geriatric patients
}

\author{
A. Cherubini ${ }^{1} \cdot$ M. L. Laroche $e^{2,3,4} \cdot$ M. Petrovic $^{5}$
}

Published online: 8 April 2021

(c) European Geriatric Medicine Society 2021

The management of pharmacological therapy in older patients is challenging in clinical practice. Older subjects experience age-related changes that influence pharmacokinetics and pharmacodynamics of drugs, they also usually have multiple diseases, i.e., multimorbidity, and are often cared for, and hence, prescribed by a number of physicians over a long period of time. Therefore, it is not surprising that polypharmacy, literally the use of multiple drugs, is extremely common in older patients and is increasing over time, at least in developed countries $[1,2]$.

Appropriate geriatric pharmacotherapy, global assessment of patients' clinical and functional parameters, and integration of skills from different healthcare professionals are needed to address the medical complexity of older adults.

In this special issue, the most relevant aspects of pharmacotherapy in older people have been addressed.

In the paper discussing drug metabolism and elimination in older people in the perspective of personalized prescription, Mangoni and Jarmuzewska review the current evidence regarding the main age-associated changes in drug metabolism and elimination and elaborate on their possible inclusion in current and future personalized prescribing tools for older patients [3]. The integration of pharmacokinetic data

\section{A. Cherubini}

A.CHERUBINI@inrca.it

1 Geriatria, Accettazione Geriatrica e Centro di ricerca per l'invecchiamento, IRCCS INRCA, Ancona, Italy

2 Centre de Pharmacovigilance, de Pharmacoépidémiologie et D'information sur les Médicaments, Centre de Biologie et de Recherche en Santé, Service de Pharmacologie, Toxicologie et Pharmacovigilance, CHU de Limoges, Limoges, France

3 Université de Limoges, INSERM 1248, Faculté de Médecine, Limoges, France

4 Université de Limoges, Unité Vie-Santé, Faculté de Médecine, Limoges, France

5 Department of Internal Medicine and Paediatrics, Section of Geriatrics, Ghent University, Ghent, Belgium into personalized prescribing remains a hypothetical construct in geriatric care. In anticipation of the inclusion of frail and complex older patients in pre- and post-marketing studies, a better understanding of the key pharmacokinetic alterations of common medications in "real-life" patients, together with the implementation of effective strategies tackling inappropriate prescribing, is likely to improve clinical outcomes and reduce healthcare utilization in the older population.

In the paper on definition, epidemiology and consequences of polypharmacy, Pazan et al. show that there is no universally accepted definition of polypharmacy [4]. While many different definitions have been used, the majority only consider the number of drugs. The most common one is based on treatment with five or more drugs. This concept is not very useful as a relevant problem with the use of multiple drugs is the frequent presence of inappropriate drugs. On this premise, it has been proposed to focus on problematic or unnecessary rather than appropriate polypharmacy [5, $6]$. The heterogeneity of definitions might also be responsible for the controversial relationship of polypharmacy with negative clinical outcomes in different studies. Nevertheless, the association of polypharmacy with geriatric conditions, such as frailty, falls, physical function and cognitive impairment as well as with hospitalization, mortality has been documented in multiple studies. WHO declared that polypharmacy is a relevant target to address to increase patient safety [7].

Cherubini et al. address the causes and consequences of underprescription of medications in older adults. They show that despite the fact that polypharmacy is widespread, there are multiple studies illustrating that many older subjects are not treated with drugs appropriate for their disease, a condition known as underprescription [8]. Among the medicines most commonly underprescribed, there are cardiovascular drugs, e.g., ace inhibitors and statins, anticoagulants and anti-osteoporosis drugs. The consequences of underprescription are relevant for patients and healthcare systems, 
in terms of higher risks of cardiovascular events, disability, hospitalization and death. While some studies showed that interventions based on the implementation of explicit criteria for underprescription, comprehensive geriatric assessment by geriatricians, as well as the involvement of a clinical pharmacist can reduce underprescription, the improvement of clinical outcomes has not yet been convincingly demonstrated. Furthermore, there is still need for high-quality research with a more in-depth analysis of the causes of underprescription that in some cases might be correct, e.g., when patients are allergic or intolerant to the drug or the lag time needed for a specific drug to be beneficial is longer than the estimated life expectancy of a patient.

Capiau et al. elaborate on a guidance for appropriate use of psychotropic drugs, as the most prescribed drug class in older people [9]. They emphasize that the appropriate use of these drugs in older people can be optimized in terms of various aspects, such as indications, contraindications, dosing, adverse drug reactions, interactions and duration of therapy. Different strategies and practical recommendations should be considered to increase the appropriate use of these medications in older people. The importance of a multifaceted approach including non-pharmacological interventions, comprehensive medication reviews, shared decision-making and close interprofessional communication and collaboration is highlighted.

In the paper on potentially inappropriate medication use among older people with dementia, Renom Guiteras discusses several aspects that are considered when patient tailoring decisions are made on the pharmacotherapy appropriateness among older people with dementia [10]. The aspects that may deserve special consideration include the prescription of psychotropic medications, for being commonly inappropriately prescribed; the presence of advanced stage of dementia, comorbidities or multimorbidity and/or frailty, as they can determine the prognosis and goals of care; the values and wishes of the person with dementia, as they may prioritize different goals of care; and medication adherence, as it may be poorer compared with persons without dementia. These individualized decisions require a comprehensive evaluation of the patient. The need for studies including representative study participants and further evidence-based guidelines are necessary to support these decisions.

Zazzara et al. address the important topic of adverse drug reactions (ADRs). ADRs are frequent in older adults and are often related to commonly used medications [11]. Certain risk factors for the occurrence of ADRs are uncontrollable, but others can be dealt with better by considering the older patients in their complexity. The authors show that approaches such as medication review, software identifying potentially inappropriate prescriptions, are sometimes disappointing in ADR prevention because they are too focused on the drugs, compared to the comprehensive geriatric assessment which demonstrates a better identification of patients at risk of ADR occurrence and also takes more into account the care objectives and the patients' needs. The authors advocate a multidimensional and holistic approach combining pharmaceutical interventions with a global evaluation of health needs and priorities to reduce the burden of ADRs in older adults.

In the next paper of this special issue, Rochon et al. focus on prescribing cascades as a phenomenon closely associated with polypharmacy [12]. The use of multiple drugs is often associated with the occurrence of side effects, which might be misinterpreted as new symptoms or clinical events and lead to the prescription of new drugs to treat them. Research in this field is still relatively new, and therefore, the prevalence and clinical relevance of prescribing cascades have not yet been systematically established. Moreover, the presence of differences related to sex and gender in the occurrence and consequences of this condition are currently unknown. To provide a better knowledge of prescribing cascades focusing specifically on sex and gender, is the aim of the iKascade project, an international research project whose rationale and design are presented here. The project involves an international team of experts led by Paula Rochon, a geriatrician and expert in gender medicine from Canada.

In the paper on fall risk-inducing drugs, van der Velde et al. point out that falls are a geriatric syndrome that often have a dramatic impact on health and the level of autonomy of older subjects [13]. Falls represent an important cause of fractures, head trauma and mortality in older subjects and, even when they do not determine immediate negative consequences, they can induce a fear of falling, leading to social isolation, immobility and, paradoxically, an increased risk of falling. Falls are typically multifactorial and several drugs, named fall risk increasing drugs (FRID), can contribute to increase the probability of experiencing a fall. The European Geriatric Medicine Society working groups produced an explicit tool to identify FRID (STOPFALL) together with guidance to support an evidence-based approach in deprescribing these drugs [14] Among FRID, there are also antidepressants, which can contribute to increase the risk of falling with different mechanisms, including sedation, delirium, hyponatraemia, orthostatic hypotension, cardiac side effects and impaired mobility. On the other hand, depression has several negative consequences in older adults and increases the risk of falling. Therefore, a careful evaluation of the individual risk benefit ratio should be made when deciding the prescription or continuation of antidepressant therapy, particularly in older people who are at increased risk of falling. When appropriate, deprescribing should be pursued according to the available evidence and specific expertise.

In the paper written by Laroche et al., the fundamentals of drug-related problems (DRPs) in older adults are put 
in perspective according to a logical process, conciliating medical and pharmaceutical approaches to better identify the causes and consequences of DRPs [15]. This process analysis is carried out at the physician, pharmacist, nurses and patient level, to understand the phenomenon of DRPs, to prevent the consequences leading to negative benefit/risk ratio of drugs, and to identify corrective actions to improve medication use among older people. This overview shows that medication errors are not the most frequent cause of DRPs. Surprisingly, substance use disorders are rarely studied in older adults and need to be investigated further because the use of psychoactive substances among older people is frequent. Finally, to prevent DRPs in older adults, improved communication between healthcare professionals, patients and their carers would ensure greater safety and effectiveness of treatments.

Beuscart et al. explain that medication reconciliation (MedRec) and review (MedRev) are complementary in preventing medication-related problems in older adults [16]. These strategies are successful because they integrate the skills of different health care professionals who have to deal with the medical complexities of older patients. However, these procedures are time-consuming and require a perfect coordination of all the different healthcare professionals. Information technology (IT) can be considered as an assistance for MedRec or MedRev, by means of an automatic data analysis. The authors insist on the paradigm shift that healthcare professionals in geriatrics must do: from a disease-centered approach to patient-centered care, communication and shared decision-making.

Spinewine et al. describe strategies and interventions to optimize medication use in nursing homes. The authors carried out a two-level analysis of the health care system [17]. For the personnel and residents, medication review, multidisciplinary work and patient-centered care are the main approaches proven to be effective in nursing homes. However, there is a large heterogeneity in the outcomes across the studies. It would be preferable to use core outcome sets focused on nursing home residents or multimorbid older adults. At the system-level, legislations and underperforming care networks are the main barriers holding up optimal implementation of procedures, with a crucial need for funding, especially in Europe.

In the paper on effectiveness of hospital pharmacists in improving drug therapy and relevant health outcomes, Delgado et al. review the current evidence regarding the effects of interventions by hospital pharmacists, individually or in collaboration with a multidisciplinary team, in terms of healthcare outcomes, effective utilization of resources and lower costs in older patients with multimorbidity and polypharmacy [18]. They conclude that so far there is no hard evidence that demonstrates the effectiveness of hospital pharmacists' interventions in older multimorbid and polymedicated patients with regard to health-related outcomes and that better-designed studies are warranted in the future to provide further insights into the effect of hospitals pharmacists interventions.

Finally, in the paper on the use of opioids in the geriatric patient at the end of life, Janssens et al. describe pharmacological treatment at the end of life, comparing use and dosage of opioids in the acute geriatric ward and palliative care unit, pointing out that achieving adequate symptom control is related to the use of opioids [19].

In this special issue, the most relevant aspects of geriatric pharmacotherapy are presented and critically discussed. The optimal management of drugs in older patients is a cornerstone of the practice of geriatric medicine and one of the most important contributions to the evolution of health care in the direction of adapting contemporary clinical practice to the specific needs of older patients. However, while the tasks are clear, i.e., tailoring drug therapy according to the personal preferences and characteristics, increase patient safety by reducing adverse drug reactions, achieve the most favorable risk benefit ratio for each patient, periodically reviewing pharmacological therapy including deprescribing, as appropriate, the implementation in practise is often difficult, particularly when health care professionals are not geriatricians or clinical pharmacists.

A research priority is, therefore, to develop and validate interventions that can be applied by healthcare professionals who are not highly skilled in geriatric pharmacotherapy. The recently published results of the SENATOR trial, a large randomized clinical trial which failed to achieve a reduction of adverse drug reactions by means of a highly sophisticated software aimed at optimizing pharmacotherapy in hospitalized older patients, confirm the persistence of this important unmet need [20].

\section{Compliance with ethical standards}

Conflict of interest The Authors declare no conflict of interest.

Ethics approval Ethical approval is not required for this manuscript.

Informed consent Informed consent is not required for this manuscript.

\section{References}

1. Qato DM, Wilder J, Schumm LP, Gillet V, Alexander GC (2016) Changes in prescription and over-the-counter medication and dietary supplement use among older adults in the United States, 2005 vs 2011. JAMA Intern Med 176(4):473-482. https://doi.org/ 10.1001/jamainternmed.2015.8581

2. Zhang N, Sundquist J, Sundquist K, Ji J (2020) An increasing trend in the prevalence of polypharmacy in Sweden: a nationwide 
register-based study. Front Pharmacol 11:326. https://doi.org/10. 3389/fphar.2020.00326

3. Mangoni AA, Jarmuzewska EA (2021) Incorporating pharmacokinetic data into personalised prescribing for older people: challenges and opportunities. Eur Geriatr Med. https://doi.org/ 10.1007/s41999-020-00437-5 (Epub ahead of print)

4. Pazan F, Wehling M (2021) Polypharmacy in older adults: a narrative review of definitions, epidemiology and consequences. Eur Geriatr Med. https://doi.org/10.1007/s41999-021-00479-3 (Epub ahead of print)

5. Scottish Government Polypharmacy Model of Care Group. Polypharmacy Guidance, Realistic Prescribing 3rd Edition, 2018. Scottish Government https://www.therapeutics.scot.nhs.uk/wpcontent/uploads/2018/04/Polypharmacy-Guidance-2018.pdf Last accessed on March 1st, 2021

6. Gutiérrez-Valencia M, Martínez-Velilla N, Vilches-Moraga A (2019) Polypharmacy in older people: time to take action. Eur Geriatr Med 10(1):1-3

7. Medication Safety in Polypharmacy. Geneva: World Health Organization 2019 (WHO/UHC/SDS/2019.11). https://apps.who. int/iris/bitstream/handle/10665/325454/WHO-UHC-SDS-2019. 11-eng.pdf?ua =1 last accessed March 1st, 2021

8. Lombardi F, Paoletti L, Carrieri B, Dell'Aquila G, Fedecostante M, Di Muzio M, Corsonello A, Lattanzio F, Cherubini A (2021) Underprescription of medications in older adults: causes, consequences and solutions-a narrative review. Eur Geriatr Med. https:// doi.org/10.1007/s41999-021-00471-x. (Epub ahead of print)

9. Capiau A, Foubert K, Somers A, Petrovic M (2021) Guidance for appropriate use of psychotropic drugs in older people. Eur Geriatr Med. https://doi.org/10.1007/s41999-020-00439-3 (Epub ahead of print)

10. Renom Guiteras A (2021) Potentially inappropriate medication among people with dementia: towards individualized decisionmaking. Eur Geriat Med (in press).

11. Zazzara MB, Palmer K, Vetrano D, Carfi A, Onder G (2021) Adverse drug reactions in older adults: a narrative review of the literature. Eur Geriat Med (in press)

12. Shelley A, Sternberg SA, Petrovic M, Onder G, Cherubini A, O’Mahony D, Gurwitz J, Pegreffi F, Mason R, Akerman J, McCarthy L, Lawson A, Li J, Wu W, Rochon P (2021) Identifying key prescribing cascades in older people (iKascade): a transnational initiative on drug safety through a sex and gender lens-rationale and design. Eur Geriat Med (in press).

13. van Poelgeest EP, Pronk A, Rhebergen D, van der Velde N (2021) Depression, antidepressants and fall risk: therapeutic dilemmas. A clinical review. Eur Geriat Med (in press)

14. Seppala LJ, Petrovic M, Ryg J, Bahat G, Topinkova E, Szczerbińska K, van der Cammen TJM, Hartikainen S, Ilhan B,
Landi F, Morrissey Y, Mair A, Gutiérrez-Valencia M, EmmelotVonk MH, Mora MÁC, Denkinger M, Crome P, Jackson SHD, Correa-Pérez A, Knol W, Soulis G, Gudmundsson A, Ziere G, Wehling M, O’Mahony D, Cherubini A, van der Velde N (2020) STOPPFall (screening tool of older persons prescriptions in older adults with high fall risk): a Delphi study by the EuGMS task and finish group on fall-risk-increasing drugs. Age Ageing 22:afaa249. https://doi.org/10.1093/ageing/afaa249.

15. Laroche ML, Thi Hong Van Ngo, Sirois C, Daveluy A, Guillaumin M, Valnet-Rabier MB, Grau M, Roux B, Merle L (2021) Mapping of drug-related problems among older adults conciliating medical and pharmaceutical approaches. Eur Geriat Med (in press).

16. Beuscart JB, Pelayo S, Robert L, Thevelin S, Marien S, Dalleur O (2021) Medication review and reconciliation in older adults. Eur Geriatr Med. https://doi.org/10.1007/s41999-021-00449-9.

17. Spinewine A, Evrard P, Hughes C (2021) Interventions to optimize medication use in nursing homes. A narrative review. Eur Geriat Med (in press).

18. Delgado-Silveira E, Vélez-Díaz-Pallarés M, Muñoz-García M, Correa-Pérez A, Álvarez-Díaz AM, Cruz-Jentoft AJ (2021) Effects of hospital pharmacist interventions on health outcomes in older polymedicated inpatients: a scoping review. Eur Geriat Med (in press).

19. Jannens W, Van Den Noortgate NJ, Piers RD (2021) Pharmacological treatment in the dying geriatric patient: describing use and dosage of opioids in the acute geriatric wards and palliative care units of 3 hospitals. Eur Geriat Med (in press).

20. O'Mahony D, Gudmundsson A, Soiza RL, Petrovic M, Jose CruzJentoft A, Cherubini A, Fordham R, Byrne S, Dahly D, Gallagher P, Lavan A, Curtin D, Dalton K, Cullinan S, Flanagan E, Shiely F, Samuelsson O, Sverrisdottir A, Subbarayan S, Vandaele L, Meireson E, Montero-Errasquin B, Rexach-Cano A, Correa Perez A, Lozano-Montoya I, Vélez-Díaz-Pallarés M, Cerenzia A, Corradi S, Soledad Cotorruelo Ferreiro M, Dimitri F, Marinelli P, Martelli G, Fong Soe Khioe R, Eustace J (2020) Prevention of adverse drug reactions in hospitalized older patients with multi-morbidity and polypharmacy: the SENATOR* randomized controlled clinical trial. Age Ageing 49(4):605-614. https://doi.org/10.1093/ageing/afaa072

Publisher's Note Springer Nature remains neutral with regard to jurisdictional claims in published maps and institutional affiliations. 